\title{
ARVino - Outdoor Augmented Reality Visualisation of Viticulture GIS Data
}

\author{
Gary R. King, Wayne Piekarski, and Bruce H. Thomas \\ Wearable Computer Laboratory \\ School of Computer and Information Science \\ University of South Australia \\ Mawson Lakes, SA, 5095, Australia \\ gary@tinmith.net,wayne@cs.unisa.edu.au,bruce.thomas@unisa.edu.au
}

\begin{abstract}
This paper describes the combination of two technologies, augmented reality and GIS, to provide a new way to visualise viticulture GIS data using outdoor mobile computers. Viticulturists use GIS to assist with accurately understanding the parameters that affect their yields and quality of the grapes from different vineyards. The ability to view this data in the field digitally would be advantageous to the viticulturist. This paper describes the ARVino system; an AR platform that was built for visualising $3 D$ data outdoors using a movable tripod-based computer. We describe the user interface, some problems that were encountered, and how the visualisation and interface were evaluated through an expert review.
\end{abstract}

\section{Introduction}

Viticulturists currently use geographic information systems (GIS) to help them accurately understand the parameters that affect their yields and quality of grapes from different vineyards. The goal of this investigation is to provide the viticulturist with the ability to intuitively view this data in the field. Our new ARVino system provides a new form of visualisation of viticulture GIS data using an outdoor augmented reality (AR) system. We worked with the expert assistance of Dr. Richard Hamilton, a senior viticulturist with Southcorp, the largest wine producer in Australia. Using our existing Tinmith AR software architecture [7], we embarked on an investigation to improve visualisation of GIS information in-situ at the vineyard. Our tripod-based mobile computer allows multiple users to observe the AR overlay on an LCD screen, as shown in Figure 1. The ARVino platform overlays the physical world with a visualisation of harvest yield and Normalised Difference Vegetation Index (NDVI) data. Desktop-based

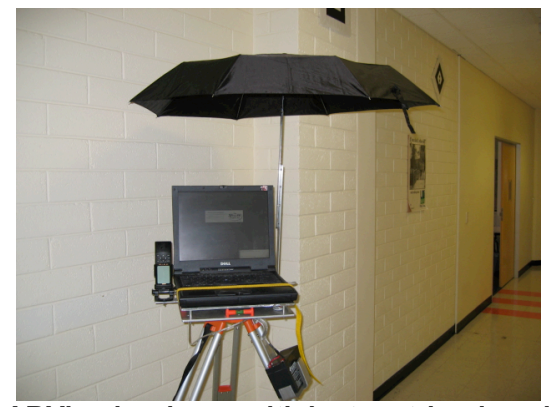

Figure 1. ARVino hardware with laptop, tripod, and umbrella
GIS software allows the viticulturists to manipulate this data to produce meaningful visualisations, but this process occurs in a traditional indoor office.

This paper presents the results of the project to date. We first provide a brief introduction to key related work. A basic overview of the ARVino system is then provided. An expert review is described and recorded results are presented. Based on the expert review, the implemented further extensions to ARVino are then described. The paper finishes with some concluding remarks.

\section{Related Work}

A number of researchers have investigated the use of virtual environments with GIS data. The ARPRISM project investigated different interface technologies for viewing GIS data, including AR, VR, and hybrid technologies [6]. The main aim of the ARPRISM project was to investigate these technologies for face-to-face collaboration, and included a prototype interface designed to allow multiple users to view and interact with geospatial data.

Ghadirian and Bishop investigated [4] an immersive environment that combined GIS-based environmental process modelling with AR viewing. The authors noted that although there is an increase in the development and use of virtual environment systems, there is still a large gap between the best virtual worlds and the real environment. The main reason put forward is that although they may have highly realistic animation there is no user interaction, or they may have some level of user control with degraded simulation.

The Augurscope [9] enabled visitors to visualise with $\mathrm{AR}$ and augmented virtuality what a historical castle looked like, and its layout in relation to the existing palace, by overlaying the real-world view and existing palace with $3 \mathrm{D}$ models. Avatars of pre-recorded actors in a medieval setting were also used to enhance the user's experience, and described various features of the 3D medieval castle. The Augurscope was built around a laptop mounted on a tripod with wheels. To conceal the keyboard from users and shield the display from sunlight, the laptop had a wooden encasing. Problems with tripod mobility and sunlight obscuring the display were noted by the authors.

The requirement to support human-to-human interactions was demonstrated in Hedley et al. [6]. Their informal trials with almost 100 users showed users liked the ability 
to see other each other in the physical world as well as in the AR model. Hedley et al. felt that this supported natural face-to-face collaboration. Users preferred the hand-held version of the Sony Glasstron HMD instead of a traditionally head worn one because it was easier to share views with others, reduced their feeling of separation from the physical world, and caused less feelings of nausea.

\section{Initial Solution}

The first objective of this investigation is to build a new AR platform that is robust enough for agricultural use. The second objective is to develop appropriate user interface techniques for this AR platform. This section will provide an overview of our initial ARVino system.

\subsection{GIS Data}

There are two types of GIS data we wish to visualise. The first is the harvest yield monitoring values, which have only fairly recently been available [3]. The other data type is the Normalised Difference Vegetation Index (NDVI), which is related to the proportion of photosynthetically absorbed radiation data derived from aerial imaging [8] . We employed the ESRI ArcGIS commercial GIS system for processing the data files, used by Southcorp. Using ESRI ArcToolbox, we convert the supplied SouthCorp shape files containing the yield and NDVI data into the Data Exchange Format (DXF) used by most CAD tools. We developed a custom utility to convert the DXF file into a VRML representation.

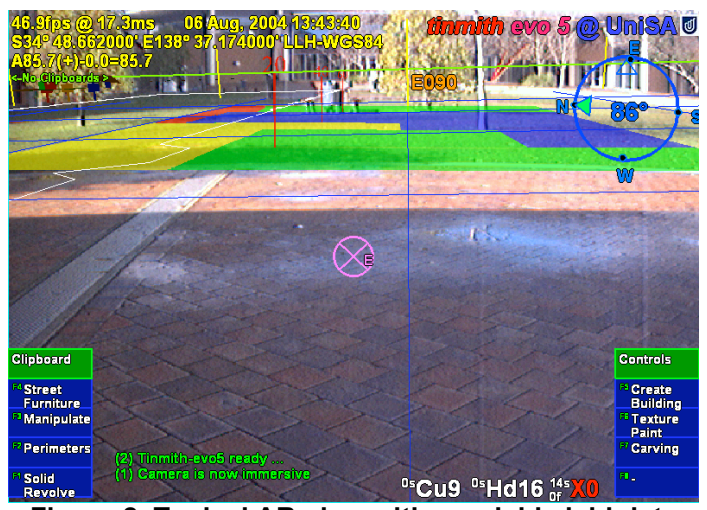

Figure 2. Typical AR view with overlaid yield data

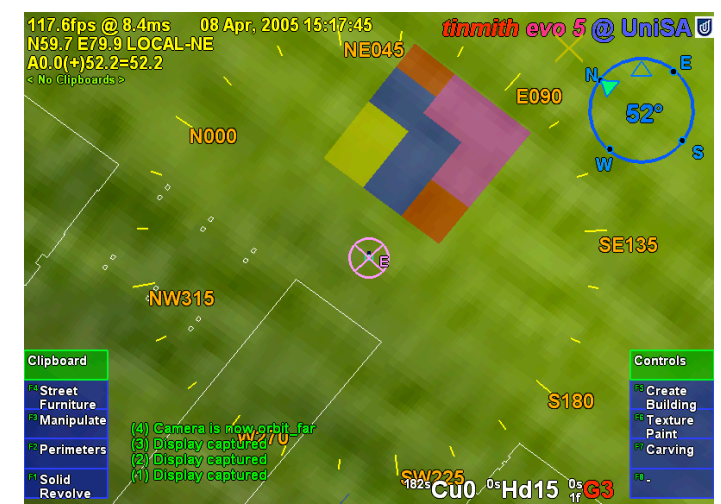

Figure 3. Top-down orbital view of yield data in VR mode

\subsection{The Platform}

We used the Tinmith software system [7] to provide the desired AR functionality. The Tinmith-evo5 software architecture supports the flexible development of mobile AR and other 3D interactive applications with limited computing resources. The AR platform is built with a non-ferrous surveyor's tripod that will not interfere with the magnetometer in our orientation sensor. We added a swivelling platform to the tripod to accommodate a 'turn-table' platform for the Dell Inspiron 8100 laptop, a firewire video camera, a Garmin 12XL 12-channel hand-held GPS unit, and a TCM2 magnetic orientation sensor. The video camera and orientation sensor are attached to the back of the LCD screen and not the base. Since the data sets are large and at long distances, the Garmin GPS unit is accurate enough. The stable tripod platform allows for a much simpler orientation sensing technology like the TCM2. The turntable is constructed from two layers of $30 \mathrm{~cm} \mathrm{x} 30 \mathrm{~cm} \mathrm{x}$ $0.5 \mathrm{~cm}$ aluminium sheet separated by $3 \mathrm{~cm}$ thin shafts producing a sandwich-type effect for all the cabling and some components within. The laptop and GPS are oriented toward the user so they can monitor the operation of the system easily. The turntable has an effective 360-degree movement, 180 degrees in either direction from a stopper. The stopper prevents the breaking of the power cable from the turntable to the battery that is mounted on the tripod base. To provide a platform which is easily made level, two mini spirit levels ( $\mathrm{X}$ and $\mathrm{Y}$ horizontal axis) are fitted to the $\mathrm{X}$ and $\mathrm{Y}$ horizontal axes for calibration.

We utilise the laptop's LCD screen, and a keyboard and touchpad mouse as input devices. This configuration allows groups of users to share the view, and able to engage and disengage easily from the display without fitting or removing any devices. The orientation of the physical world observed and the AR overlayed GIS data can be physically changed by turning the turntable about the vertical axis. The pitch of the view is achievable by simply changing the angle of the laptop screen with the camera and orientation sensor attached to the rear.

An example AR overlay is shown in Figure 2. However, the Tinmith 3D renderer also supports external virtual reality (VR) viewpoints. This provides a different perspective of the data by allowing the user to break the AR constraint of registration and leave their physical location. The video overlay is replaced with a cloud box and ground plane. The user can orbit around their location by physically adjusting the heading and pitch of the display, and change the VR view-point via the mouse and arrow keys. An example of the VR view is shown in Figure 3.

\section{Field Trial}

We conducted an expert review of ARVino. Expert reviews have been shown to be useful form of evaluation, as such a review comes from a position of knowledge [2]. The methodology entails reviews by experts in a related field to the problem and who are not directly involved in 
the project. We conducted an unstructured interview to elicit qualitative responses from the expert reviewer. The intention of the expert review conducted here was to receive feedback regarding the cognitive value of the various visualisations from the point of view of a "visualisation expert". We conducted the review with Dr. Richard Hamilton, a senior Southcorp viticulturist, as an hour-long interview. Initially, the expert was provided an overview of the ARVino system, and he was shown a set of the visualisations with the intention for him to provide qualitative feedback. The expert was then able to interact with the visualisations and provide feedback. The trial with Dr. Hamilton was conducted at our university campus. Unfortunately, real viticulture data was not available at the time of testing, but a number of simulated NDVI and water content data files were created to represent information pertaining to a grassed area of the campus.

Dr. Hamilton provided very positive comments about the system. He noted the ability of multiple people to view the GIS data in-situ was quite advantageous, as groups of up to five people typically discussed issues in the field at one time. Overall, he felt the system provided better visualisation for the viticulturist than available with traditional methods. During the field trials he noted that transparency and clarity of objects, sunlight on the display, and the long-flat viewing problem (discussed later) may all affect the final view. These three problems tend to compound in the final view.

\subsection{The Transparency Problem}

Transparency of virtual objects is employed to allow the viewing of the entire visible physical world when solid $3 \mathrm{D}$ objects are overlaid, and providing the feeling of blending between the physical world and the virtual objects. It proved difficult to balance between the ability to see clearly the virtual objects and view the physical world. Direct sunlight while viewing outdoors exaggerates this problem by further reducing the contrast of the display.

\subsection{The Long-Flat View Problem}

The long-flat view problem is caused by viewing flat virtual objects on the ground at a distance. Depth perspective causes far away objects to be small and hard to see. Figure 4 depicts how visual features are harder to view at more oblique angles, and how an object from a 40 degree raised position is much easier to view than from only a few degrees above ground level. This figure demonstrates the problem found when viewing vineyard data on a flat paddock, as is the case in many Australian vineyards.

\subsection{The Sunlight Problem}

When viewing computer screens outdoors in bright sunlight, the display can be almost unreadable. Attempts to minimise this problem include the use of shields [5] [9] and dark translucent plastic [1] to protect the screen from ambient light. However both of these had only minimal effect since the outdoor environment is still very bright compared to the display itself.

\section{Solutions}

After the field trial, we investigated and implemented a number of solutions to the previously mentioned problems of colour, long-flat viewing, and sunlight. We added two additional features: aerial photos as ground textures for VR viewing, and collaboration techniques.

\subsection{Colours}

Dr. Hamilton reported the number of colours we currently used was excessive, and that three or four colours would be ample since the viticulturists usually only divide crops into high, medium, or low yields [3]. Experimenting with the colours and the lighting properties of the VRML objects minimised the transparency problem. It was found that best results were obtained by using bright simple colours such as pure red, green, and blue, and mixes such as cyan, magenta, and yellow. The $3 \mathrm{D}$ renderer was configured to $100 \%$ ambient lighting to remove any possibility of shading which could make the objects darker.

\subsection{Long-Flat View}

We employed VRML billboards and marker posts to help establish distances from the viewer in relation to the data. Figure 5 depicts a set of billboards and marker posts at varying distances. VRML billboards are rendered so text or other information is always oriented towards the user. The distance markers give the user a reference in metres from a known distinguishable physical object such as a boundary fence. The markers are linearly placed, cutting across the virtual GIS data into the distance.

We explored the use of a second camera to capture the view of the physical world from a higher perspective. By placing the camera on a pole 1.5 metres above the first, the view could be improved, and simulate the user's experience from the top of a ladder. With the viewing height changing from $1.5 \mathrm{~m}$ to $3.0 \mathrm{~m}$, the viewing angle would be double and provide a greater perception of depth and clarity from the data. This form of remote AR viewing removes the user from a direct see-through display to an indirect see-through one. We plan on investigating this periscope-like technique further.

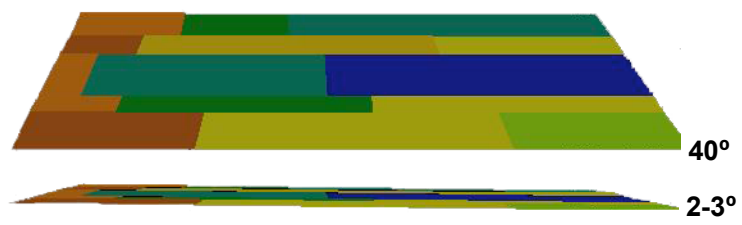

Figure 4. Flat View Problem

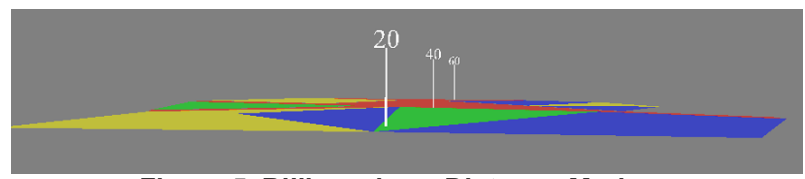

Figure 5. Billboards as Distance Markers 


\subsection{Sunlight}

The use of bright colours and full ambient lighting with the VRML objects helped to enhance their visual quality on the laptop display, but we discovered users with brightly coloured clothing reflected light onto the screen and into their eyes. By wearing dark clothing the reflections were minimised significantly, and the use of a peaked baseball cap additionally helped.. Another simple fix was the addition of a mounting bracket to hold a small personal umbrella that provides shade over the display.

\subsection{Use of Aerial Photos}

The VR view allows the user to detach from the physical world and view the information using an orbital-style view. We found this sometimes disorients the user as to where the data is relative to the physical world. We have performed testing with aerial photography of the area as a texture for the VR view ground plane. Figure 6 shows an example of some yield data overlaid transparently onto an aerial photograph. Features in the aerial image allow the user to register the VR data with the physical world.

\subsection{Collaboration}

Based on the comments from Dr. Hamilton, we have performed investigation into the integration of a wearable AR system with a HMD in continuous communication with the ARVino tripod system. We have tested our existing Tinmith software architecture [7] in supporting the sharing of data between multiple systems both indoors and outdoors. By leveraging this technology we would be able to support a mobile expert tracked in the vineyard while others watch from the tripod system. A wireless 802.11 base station provides the communication between the systems and allows the sharing of position and orientation information, as well as the 3D GIS data. To support effective collaboration, we have tested bi-directional audio transmission using a GSM codec over the wireless network. Both the mobile users, and users at the tripod, are able to give verbal information back and forth.

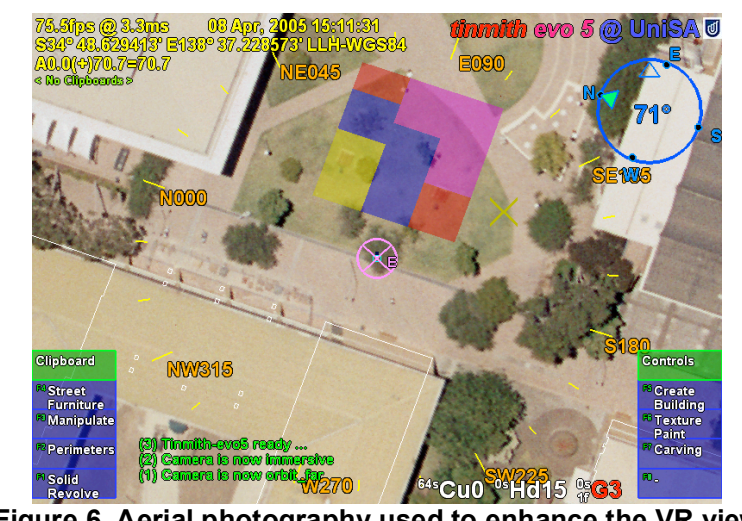

Figure 6. Aerial photography used to enhance the VR view

\section{Conclusion}

The research described in this paper has shown that GIS data, vital to the modern viticulturist, can be visualised effectively on an outdoor AR system. We described the development of our ARVino platform using existing AR software and hardware, presented in a custom-built tripod configuration. Trial and evaluation of GIS data representations on the AR platform were conducted, and the associated problems discussed. These problems included the long-flat view problem, transparency, and display contrast. The long-flat view problem is likely to be encountered by anyone attempting to view agricultural or land data over flat ground, and our periscope and VR views attempt to provide a solution. By using saturated colours, dark clothing, and an umbrella, the display quality can be improved significantly. Expert opinions from a Southcorp viticulturist have guided the development of our visualisations and recognised advantages that may be used to assist viticulturists in the future.

\section{References}

[1] Azuma, R., Hoff, B., Neely, H., and Sarfaty, R. A Motion-Stabilized Outdoor Augmented Reality System. In Proceedings of IEEE Virtual Reality '99, Houston, Tx, Mar 1999.

[2] Booth, P. An Introduction to Human-Computer Interaction. Lawrence Erlbaum Associates, 1989.

[3] Bramley, R. G. V. and Hamilton, R. P. Understanding variability in winegrape production systems. Australian Journal of Grape and Wine Research, No. 10, pp 32-45, 2004.

[4] Ghadirian, P. and Bishop, I. D. Composition of Augmented Reality and GIS To Visualize Environmental Changes. In Joint AURISA and Institution of Surveyors Conference, Adelaide,SA, Nov 2004.

[5] Gleue, T. and Dähne, P. Design and Implementation of a Mobile Device for Outdoor Augmented Reality in the ARCHEOGUIDE Project. In VAST 2001 - Virtual Reality, Archeology, and Cultural Heritage International Symposium, Athens, Greece, Nov 2001.

[6] Hedley, R. H., Billinghurst, M., Postner, L., May, R., and Kato, H. Explorations in the Use of Augmented Reality for Geographic Visualization. Presence: Teleoperators and Virtual Environments, Vol. 11, No. 2, pp 119-133, 2002.

[7] Piekarski, W. and Thomas, B. H. An Object-Oriented Software Architecture for 3D Mixed Reality Applications. In 2nd Int'l Symposium on Mixed and Augmented Reality, Tokyo, Japan, Oct 2003.

[8] Richard, Y. and POCCARD, I. A statistical study of NDVI sensitivity to seasonal and interannual rainfall variations in southern Africa. International Journal of Remote Sensing, Vol. 19, No. 15, pp 2907-20, 1998.

[9] Schnadelbach, H. et al. The Augurscope: A Mixed Reality Interface for Outdoors. In CHI, Minneapolis, Mn, Apr 2002. 